\title{
Oven Fluid Analysis and Parameter Optimization for Gravure Printing Machine YF93
}

\author{
Qiaoxia Gao ${ }^{1, a}$, Xianwen Shen ${ }^{2, b^{*}}$ \\ ${ }^{1}$ Chongqing Business Vocational College in Chongqing, China \\ ${ }^{2}$ Chongqing University in Chongqing, China \\ agaoqiaoxia014@126.com, bshenxianwen0528@163.com
}

\begin{abstract}
Keywords: gravure printing machine; drying oven; computational fluid dynamics; velocity field Abstract. Gravure printing machine Oven hot air dynamic performance and structural demensions directly affect the drying effect of print. In this paper, fluid mechanics is used to analyze kinematic and dynamic behavior of hot air flow in the oven of a gravure printing machine. The CFD method was adopted to do dynamic simulation and quantitative analysis of hot air flow in existing ovens. The simulation results and test data showed that it is feasible to use the computer simulation method to do fluid dynamic analysis of an oven structure; through calculations and simulation analysis, the oven inner working states can be clarified, the key processing parameters can be determined and optimized. The research results provided a theoretical basis for the improvement and optimization of gravure printing machine oven structure.
\end{abstract}

\section{Introduction}

In recent years, domestic gravure printing machine manufacturing technology has greatly improved on the whole, but the printing speed, print quality is generally low. Apart from limited machinery precision and control precision, drying process becomes the important bottleneck of printing speed, which has aroused wide attention from experts[1-7]. Jimei Wu etc.[4] analyzed traditional oven model and built the relationship betwenm heat wind output parameters and input parameters. Qingming Huang etc.[8]designed the programmable temperature control system based on self-adaptive fuzzy PID algorithm as drying oven temperature Characteristics of nonlinear, time-varying and hysteresis.

Many factors force drying system to optimize and improve, such as print quality, energy consumption, environmental protection, multicolor, the development trend of high performance and customer enterprise profit maximization. In this paper, the oven YF93 is analyzed by the CFD simulation method, and the oven working condition and the key process parameters are obtained.

\section{Heat Wind Analysis Method[10-13]}

The difference between inflow and outflow mass through control surface is equal to infinitesimal body internal mass Increment:

$$
\frac{\partial \rho}{\partial t}+u \frac{\partial(\rho u)}{\partial x}+v \frac{\partial(\rho v)}{\partial y}+w \frac{\partial(\rho w)}{\partial z}=0
$$

where $\rho$ is the fluid density, $u, v$ and $w$ is the fluid speed in the $x, y$ and $z$ direction respectively.

Unit volume fluid momentum rate is equal to the sum of all external forces:

$$
\rho \frac{\mathrm{DV}}{\mathrm{D} t}=\rho \mathbf{F}+\nabla \cdot \mathbf{P}
$$

where $\rho$ is the fluid density, $\mathbf{V}$ is the fluid speed, $\mathbf{F}$ is the mass force, $\nabla$ is the hamiltonian operator, $\mathbf{P}$ is the stress tensor, $\frac{\mathrm{DV}}{\mathrm{D} t}$ is the speed variation percentage.

In the flow of the fluid system, the total power change rate is equal to the external force power on the system and the sum of external heat transfer power of the system : 


$$
\rho c_{V} \frac{D T}{D t}=\rho q+k \nabla^{2} T+\Phi
$$

where $\rho$ is the fluid density, $k$ is the coefficient of heat conduction, $q$ is the heat source, $c_{V}$ is the heat capacity at constant volume, $\Phi$ is the dissipative function, $D T / D t$ is the temperature derivative.

\section{Drying Oven Model and Simulation Details}

YF93 oven structure is shown in Fig.1. On the basis of the engineering design drawings, the model is built by $3 \mathrm{~d}$ design software Pro/E.
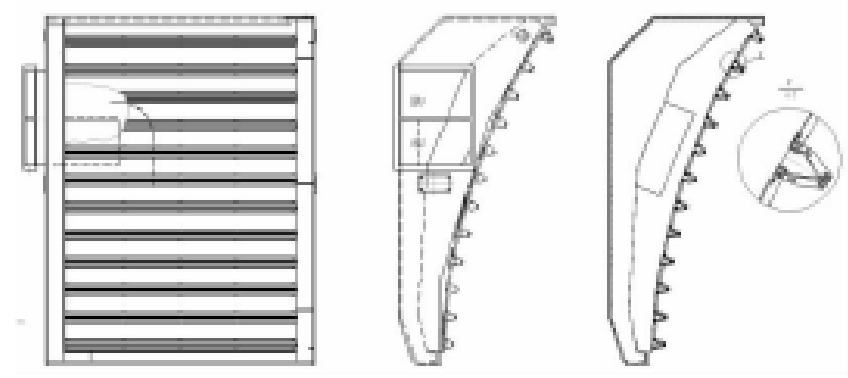

Fig.1 Oven structure 3D model

Under the premise of guaranteeing the oven main structures, it is made some appropriate simplified applications. Size is determined by the design drawing. Main size settings include the total length of tuyere, outlet width, outlet angle and outlet height, and the model is shown in figure1. As the analysis needs, 12 tuyeres are numbered from top to bottom.

In this paper, hot air is blowed from the inlet into dry Box, flows inside the oven cavity and sprays to substrates from the 12 nozzles, then returns to the bellows from two sides, discharge through the exhaust outlet.

According to the flow characteristics of hot air, unstructured quadrilateral grid and three angle grid are used in the designated surface meshing area, the tetrahedron grid in the body of the division, hexahedron, wedge, vertebral blended grid in the special places. The total number of model grid is 4 999871.
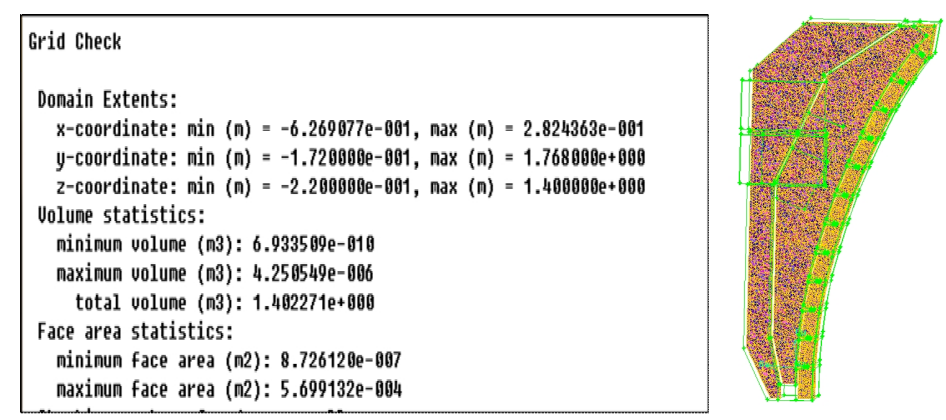

Fig. 2 Oven mesh check

Inspection on drying box grid in the FLUENT software, the result is shown in Fig.2. It is clear that the drying oven grid minimum volume is not negative, so calculation can work smoothly.

In order to judge the hot air flow is laminar or turbulent, hot wind Reynolds number is to be calculated. By the calculation, the hot air oven nozzle Reynolds number is 322730, greater than the critical Reynolds number 4000 . So it is turbulent state, should be calculated by the turbulence steady $\kappa-\varepsilon$ model. the model of $\kappa=0.391, \varepsilon=1.86$.

According to working condition and the technical documents, wall boundary conditions are as follows:

1) inlet boundary condition is set to velocity-inlet $(15.5556 \mathrm{~m} / \mathrm{s})$;

2) export boundary condition is set to pressure-export(-450Pa);

3 ) wall boundary conditions is set to the convective heat transfer wall. 


\section{Numerical Simulation Results and Discussion}

The hot wind flow trace is composed of a number of different particle curve at the same time. Every point's tangent direction on the curve parallels to the fluid particle movement direction. Under stationary flow, the shape of the trace does not change over time, and fluid particle trace map coincides with trajectory map. Studying the motion of hot air inside the oven needs to analyze particle trace.
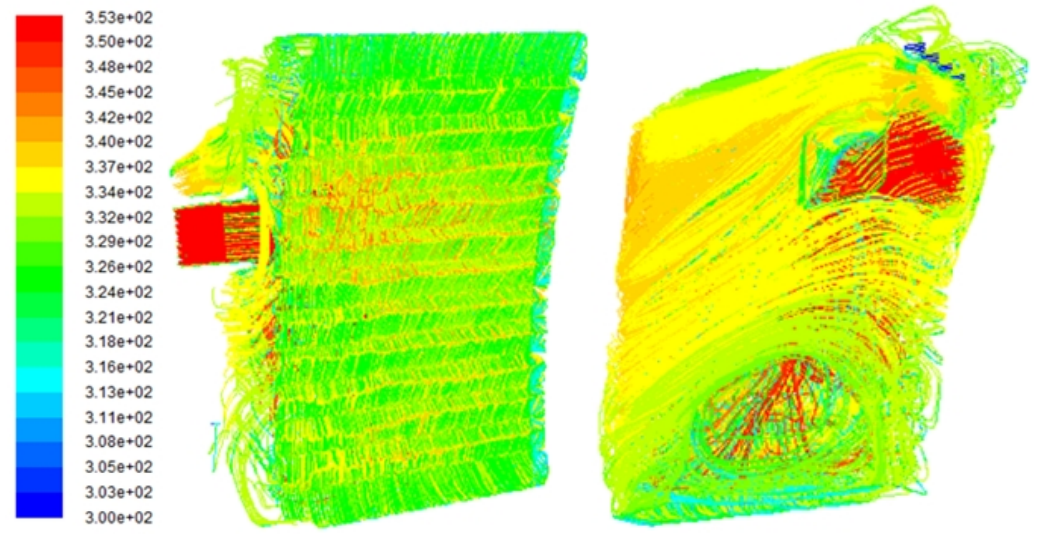

Fig. 3 Oven overall positive and back path lines

Oven flow trace chart is shown in Fig.3. The color of trace shows that hot air starts from the air inlet into the oven, the initial temperature of $353 \mathrm{k}$, through the tuyeres, and is blew to the back box. The change of the trace color reflects the change of the hot wind temperature, which refers to the color bar on the left.

In the temperature field analysis, the inlet air temperature is set as $353 \mathrm{k}$. After hot air reached steady state, oven temperature field distribution and the back box temperature field distribution are shown in Fig.4 .
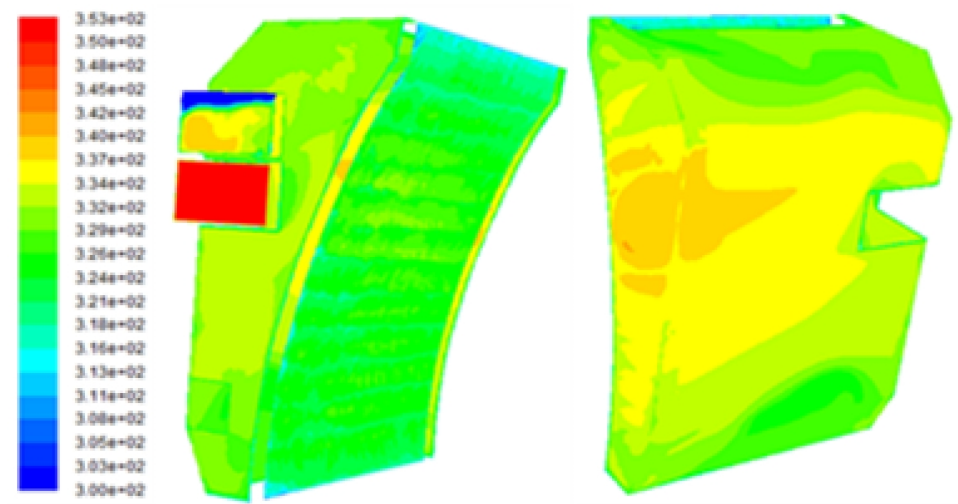

Fig.4 Oven overall temperature distribution

As shown in Fig.4, different colors represent different temperature of hot air, air inlet being red, the temperature $353 \mathrm{k}$. The back box being yellow and blue, its temperature ranges between $321 \mathrm{k}$ to $337 \mathrm{k}$, and high temperature in the middle, low temperature in the top and bottom. From the right of Fig.4, temperature is high in the opposite of hot air inlet .
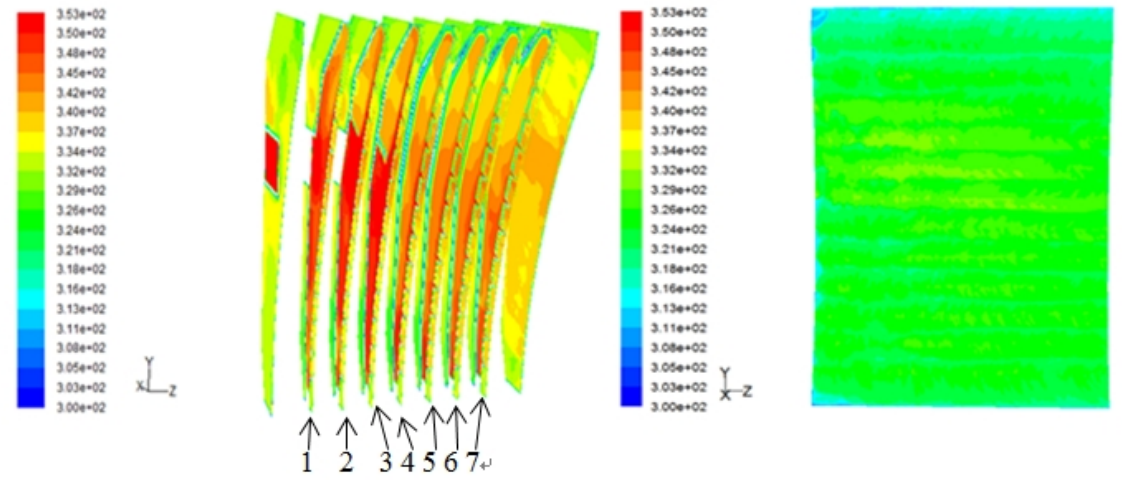

Fig.5 Oven section and substrate temperature distribution 
As can be seen from the left of Fig.5, the temperature of slices at position 1, 2, 3 is higher. Because the hot air from the air inlet into the above three positions doesn't touch the guide plate. Namely it doesn't cause the loss of energy, so its temperature keeps the hot air initial temperature by and large. By slices at position 4, 5, the temperature of the hot air begins to decline, because the hot air energy reduces after touching the guide plate; By slices at position 6,7, temperature drops further, because hot air path extends. The back box temperature is yellow and blue, high temperature in the middle, low temperature in the top and bottom.
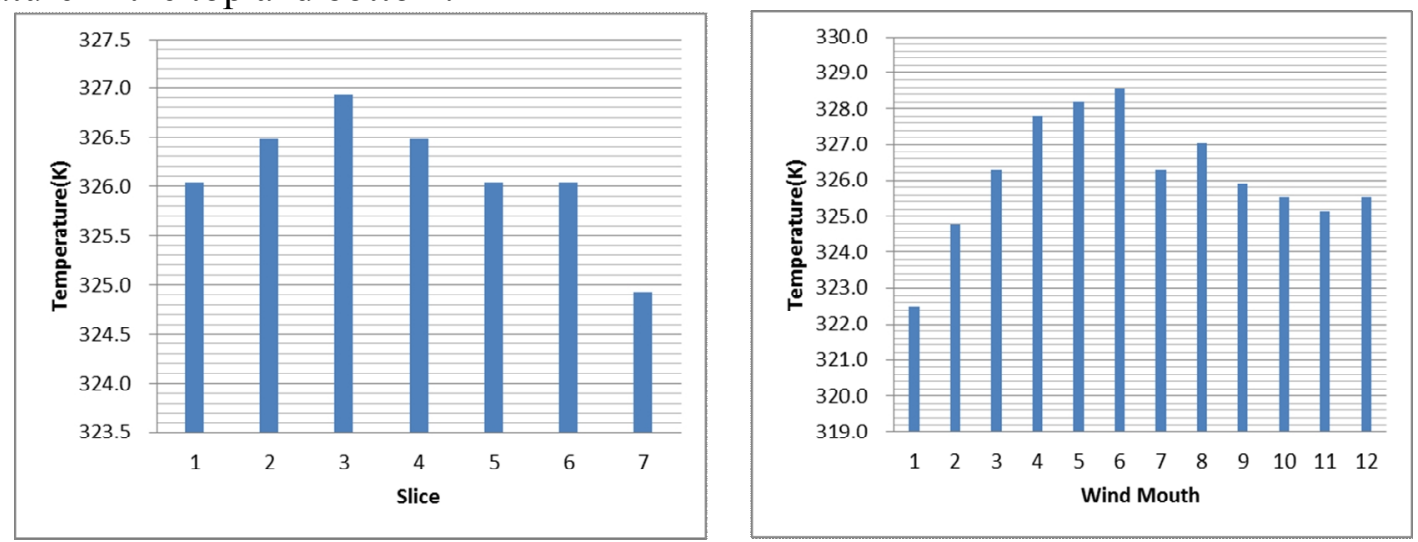

Fig. 6 Average temperature histogram

As shown in Fig.6, from 1 to 12, wind mouth temperature first rises again fells. The back box temperature is high in the middle, both sides low in order.

\section{Conclusions}

In this paper, the hot air condition analysis and parameters optimization help to improve printing quality and dry effect, the conclusion is follows:

(1)The air oven fluid domain was set up by the oven integral structure. The RNG model was built on the base of the drying oven hot air turbulence properties, and boundary conditions and parameters were set.

(2)The hot air flow pathlines of the whole body and each tuyere were obtained by the simulation analysis, which could show directly wind flow Situation in the oven and tuyere's place.

(3) The research of hot conditions and parameters in the drying oven provided references for the oven drying devices of improvement and optimization in the design.

\section{References}

[1] Fuyin Ma, Guoping Yang, Weiwei Wu. Fluid field analysis for a hydraulic impactor[J]. Journal of Vibration and Shock, 2012, 31(12): 73-78. In Chinese

[2] Wei Han , Rennian Li , Rui Yang, et al . Redesign of mechanically agitated flotation machine based on interior flow fields simulation[J]. Journal of Mechanical Engineering, 2009, 45(12): 84-88. In Chinese

[3] Xinkun Wang, Wenbo Xu, Kun Zhang, et al. Numerical simulation and design method of hot air for porous pipe based on CFD[J]. Journal of Drainage and Irrigation Machinery Engineering, 2011, 29(1): 82-86. In Chinese

[4] Jimei $\mathrm{Wu}$, Zonglei Xu, Yunchun Chen, et al. Fluid dynamic analysis and parametric optimization for drying-oven of a gravure printing machine[J]. Journal of Vibration and Shock, 2012, 31(6): 53-57. In Chinese

[5] Chow P, Cross M, Pericleous K. A natural extension of the conventional finite volume method into polygonal unstructured meshes for CFD application[J]. App. Math. Modelling, 1996, 20(2): 170-183.

[6] Van Doormal J P, Raithby G D. Enhancement of the simple method for predicting incompressible 
fluid flows[J]. Numerical Heat Transfer, 1984, 7(2): 147-163 .

[7] Cao X W, Wang L Y, Lin Z H. Air-drying models for new-built offshore gas pipelines[J]. Petroleum Science, 2005, 29(4): 46-51.

[8] Qingming Hang, Fangyuan Chen, Nengsheng Bao, et al. Research and design for temperature optimized control sestem of drying oven to gravure press[J]. Journal of Informationization of the Manufacturing, 2008, 11: 72-74. In Chinese

[9] Bruno Eck.Fans. Design and Operation of Centrifugal, Axial-Flow and cross-Flow Fans[M]. London: Pergamon Press LTD,1973.

[10] S.Kakac, A.E.Bergles, F.Mayinger. Heat Exchangers-Thermal-Hydraulic Fundamentals and Design[M]. London: McGraw-Hill, 1980.

[11] Anderson J.D. Computational Fluid Dynamics: the basics with applications[M]. New York: McGraw-Hill, 1995.

[12]Versteeg H K, Malalasekera W. An Introduction to Computational Fluid Dynamics: the finite volume method [M]. England: Longman Group Ltd, 1995. 